\title{
MODELLING A SOLAR FLARE FROM X-UV AND RADIO OBSERVATIONS
}

\author{
F. CHIUDERI DRAGO \\ Department of Astronomy and Space Science, University of Florence \\ Largo Enrico Fermi 5 - 50125 Florence, Italy
}

\author{
B.C. MONSIGNORI FOSSI \\ Arcetri Astrophysical Observatory \\ Largo Enrico Fermi 5 - 50125 Florence, Italy
}

A slow evolving flaring loop has been observed by the UVSP, XRP and HXIS instruments on board SMM on June 10,1980. Simultaneous radio observations from Toyokawa (Japan) are also available. The former instruments have an angular resolution ranging from 3 " to 30 " which allows the determination of the loop structure. The XRP:FCS rasters permit to analyze the flare only during three time intervals of about 10 minutes each.

It appears that the observations cannot be accounted for by a single loop although with a variable temperature and pressure. The presence of a hot and less dense isothermal plasma is necessary to explain the harder emission (HXIS).

$\mathrm{X}$-UV data are very well reproduced using a 2 component model consisting of a loop of constant section in which the differential emission measure varies as power law of the temperature and a hot isothermal plasma $\left(T \cong 210^{7} \mathrm{~K}\right)$ component. A full model of the loop is deduced from continuity, momentum and perfect gas law equations together with the best fit of the differential emission measure. Considering the very slow flux variation, a stationary state during each of the three time intervals is assumed. The temperature, density and velocity profiles are deduced (Chiuderi Drago and Monsignori Fossi, 1990).

The model is then checked with radio data. The comparison between computed and observed fluxes shows that they agree within a factor 2 at all frequencies.

An estimate of the total energy input in the hot component and of the energy deposition along the loop is also given. In this latter the heating function can be approximated with a $\mathrm{T}^{-2}$ decay above $\mathrm{T} \cong 2.510^{5} \mathrm{~K}$, while, at lower temperature, the heating presents some fluctuations due to the trend of the radiative losses. The total energy input in the loop increases from $2.810^{27} \mathrm{erg} \mathrm{s}^{-1}$ to $7.510^{27} \mathrm{erg} \mathrm{s}^{-1}$ from the beginning to the maximum of the flare.

A comparison with the results relative to the hot component shows that the amount of energy that must be supplied to this latter is negligible with respect to that necessary to the loop.

\section{REFERENCES}

Chiuderi Drago, F. and Monsignori Fossi, B.C.: 1990, in preparation.

E. R. Priest and V. Krishan (eds.), Basic Plasma Processes on the Sun, 454.

(c) 1990 IAU. Printed in the Netherlands. 